"This accepted author manuscript is copyrighted and published by Elsevier. It is posted here by agreement between Elsevier and MTA. The definitive version of the text was subsequently published in [PHYSICA A - STATISTICAL MECHANICS AND ITS APPLICATIONS (ISSN: 0378-4371) 448: pp. 2129. (2016), http://dx.doi.org/10.1016/j.physa.2015.12.095]. Available under license CC-BY-NC-ND." 


\section{Accepted Manuscript}

Multifractal detrended fluctuation analysis of Pannonian earthquake magnitude series

Luciano telesca, Laszlo toth

PII:

$$
\text { S0378-4371(15)01123-1 }
$$

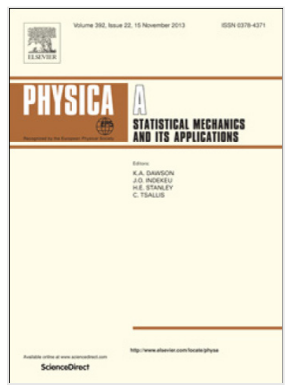

DOI: $\quad$ http://dx.doi.org/10.1016/j.physa.2015.12.095

Reference: PHYSA 16721

To appear in: Physica A

Received date: 6 June 2015

Revised date: 21 October 2015

Please cite this article as: L. telesca, L. toth, Multifractal detrended fluctuation analysis of Pannonian earthquake magnitude series, Physica A (2015),

http://dx.doi.org/10.1016/j.physa.2015.12.095

This is a PDF file of an unedited manuscript that has been accepted for publication. As a service to our customers we are providing this early version of the manuscript. The manuscript will undergo copyediting, typesetting, and review of the resulting proof before it is published in its final form. Please note that during the production process errors may be discovered which could affect the content, and all legal disclaimers that apply to the journal pertain. 


\section{Highlights}

1) The magnitudes series of the Pannonian shallow earthquakes are weakly persistent.

2) The magnitude series of the Pannonian deep events are almost uncorrelated.

3) The multifractal spectrum of deep catalogue is wider than that of the shallow one.

4) The deep series has a wider multifractal spectrum and right-skewed 


\title{
Multifractal detrended fluctuation analysis of Pannonian earthquake magnitude
}

\section{series}

LUCIANO TELESCA ${ }^{1(*)}$, LASZLO TOTH $^{2}$

${ }^{1}$ National Research Council, Institute of Methodologies for Environmental Analysis - C.da S.Loja, 85050 Tito, Italy

${ }^{2}$ Kövesligethy Radó Seismological Observatory, MTA CSFK GGI, Meredek u. 18, 1112 Budapest, Hungary

* Corresponding author: tel. +39-0971-427277, fax +39-0971-427271, email:

$\underline{\text { luciano.telesca@imaa.cnr.it }}$

\begin{abstract}
The multifractality of the series of magnitudes of the earthquakes occurred in Pannonia region from 2002 to 2012 has been investigated. The shallow (depth less than $40 \mathrm{~km}$ ) and deep (depth larger than $70 \mathrm{~km}$ ) seismic catalogues were analysed by using the multifractal detrended fluctuation analysis. The shallow and deep catalogues are characterized by different multifractal properties: i) the magnitudes of the shallow events are weakly persistent, while those of the deep ones are almost uncorrelated; ii) the deep catalogue is more multifractal than the shallow one; iii) the magnitudes of the deep catalogue are characterized by a right-skewed multifractal spectrum, while that of the shallow magnitude is rather symmetric; iv) a direct relationship between the b-value of the Gutenberg-Richter law and the multifractality of the magnitudes is suggested.
\end{abstract}


Keywords: Seismicity; Multifractal detrended fluctuation analysis

\section{Introduction}

Earthquake sequences have been widely investigated in order to detect fractal/multifractal features in the their space and time domain (Telesca et al., 2001; Telesca et al., 2002a; 2002b; Telesca and Lapenna, 2006; Telesca et al., 2012a, 2012b; Chamoli and Yadav, 2013). On the contrary, the investigation of the fractality/multifractality of earthquakes in magnitude domain has not been extensively approached so far. Lennartz et al. (2008) analysed the long-range correlation properties in the magnitude sequences of earthquakes occurred in Northern and Southern California by using the detrended fluctuation analysis (DFA), revealing that long-term memory exists in the seismic activity manifested in the temporal fluctuations of magnitudes. Varotsos et al. (2012) applied the DFA to the magnitude sequence of California seismicity between 1973 and 2003, and detected a change in the long-range temporal correlations between earthquake magnitudes from a weak persistence (DFA exponent $\sim 0.6$ ) to anti-persistence (DFA exponent even lower than 0.5) prior the occurrence of the largest events. Aggarwal et al. (2015) analysed the sequence of magnitudes of the earthquakes occurred in Kachchh area (Gujarat, Western India) from 2003 to 2012, finding that the aftershock-depleted catalogue is more multifractal and also more persistent than the whole catalogue, indicating that aftershocks contribute to increase the homogeneity and the randomness of the magnitude sequence of the whole seismicity.

In this study, we investigate the magnitude sequences of earthquakes occurred in the Pannonian Basin, considering separately the shallow and the deep seismicity, whose multifractality has not been studied so far. 


\section{Data}

The Pannonian Basin, located in northern sector of the central Mediterranean region, is bounded on the north to the east by the Carpathian mountain belt, on the south by the Dinarides mountain belt and on the west by the Eastern Alps. Recently, the area, tectonically rather complicated, has been deeply investigated (Bada et al., 2007).

The seismic activity in the region is less intense than that occurring in the peripheral areas; nevertheless, the analysis of the distribution of the total seismic energy release suggests that the basin is currently affected by deformation. The seismicity is shallow (earthquakes whose hypocental depth is within the top $30-40 \mathrm{~km}$ of the earth's crust) in the entire region, except for the Vrancea zone where the events occur at larger depth, between approximately 70 and $160 \mathrm{~km}$ (Fig. 1). From the analysis of the focal mechanism solutions it is deduced that strike-slip and thrust faulting are almost exclusive in the Southern Alps and in the Dinarides, and are exclusively strike-slip type in the Eastern Alps and Western Carpathians. In the Pannonian Basin, thrust and strike-slip faulting seems to dominate, but Vrancea events occur in a compressive regime with thrust tectonics. (Tóth et al., 2002)

The Pannonian earthquake catalogue has been compiled, comprising both historical and instrumental seismicity within a region delimited by $44.0-50.0 \mathrm{~N}$ latitude and $13.0-28.0 \mathrm{E}$ longitude mainly for the seismic hazard assessment of nuclear power plant sites. A "quasi homogenous" earthquake catalogue was obtained converting the different magnitude scales into moment magnitude (Mw). The Pannonian catalogue spans from 1501 to 2012. The application of the Reasenberg's (1985) declustering method has eliminated from the catalogue all the aftershocks leading to an earthquake catalogue comprising "independent" events. The completeness magnitude of the Pannonian catalogue decreases with the time, being 6.0 since 1500, 5.8 since 1601, 5.3 since 1701, 4.7 since 1801 and 3.5 since 1881 . These data jointly used with stress data derived from focal mechanism solutions for individual earthquakes, a 
relatively strong basis for evaluating seismic sources and seismotectonic models have been obtained for the area inside the Pannonian Basin and around (Tóth et al., 2006).

\section{The multifractal detrended fluctuation analysis}

The multifractal detrended fluctuation analysis (MF-DFA) (Kantelhardt et al., 2002) is a very effective technique to detect multifractality in nonstationary tim series. It is an extension of the detrended fluctuation analysis. If $x(i)$, with $i=1,2, \ldots, N$ is a time series, where $N$ indicates its length, firstly the socalled "trajectory" or "profile" is constructed by integration after removing its average $x_{\text {ave }}$

$y(i)=\sum_{k=1}^{i}\left[x(k)-x_{\text {ave }}\right]$

The "profile" is sub-divided into $N_{S}=\operatorname{int}(N / s)$ non-overlapping windows of equal length $s$. Since the length $N$ of the series may not be an integer multiple of the window size $s$, and a short part of the profile $y(i)$ at the end may be disregarded by the procedure, the sub-division is performed also starting from the opposite end, obtaining a total of $2 N_{S}$ segments. A polynomial of degree $m$ fits the profile in each of the $2 N_{S}$ windows and the variance is calculated by using following formula:

$F^{2}(s, v)=\frac{1}{s} \sum_{i=1}^{s}\left\{y[(v-1) s+i]-y_{v}(i)\right\}^{2}$

for each segment $v, v=1, . ., N_{S}$ and

$F^{2}(s, v)=\frac{1}{s} \sum_{i=1}^{s}\left\{y\left[N-\left(v-N_{s}\right) s+i\right]-y_{v}(i)\right\}^{2}$

for $v=N_{S+1}, . ., 2 N_{S}$. Here, $y_{v}(i)$ is the fitting polynomial in segment $v$. Then, averaging over all segments the $q$-th order fluctuation function is computed

$$
F_{q}(s)=\left\{\frac{1}{2 N_{S}} \sum_{v=1}^{2 N_{S}}\left[F^{2}(s, v)\right]^{\frac{q}{2}}\right\}^{\frac{1}{q}}
$$


where, in general, the index variable $q$ can take any real value except zero. The parameter $q$ enhances the small fluctuations if negative, otherwise, the large ones if positive. $F_{q}(s)$ will increase with increasing $s$ and from $F_{q}(s)$ versus $s$, plotted in bilogarithmic scales, for each value of $q$, we get the scaling in the fluctuation function. If the $x_{i}$ is long-range power-law correlated, $F_{q}(s)$ increases for large values of $s$ as a power-law

$F_{q}(s) \approx s^{h_{q}}$

The value $h_{0}$ corresponds to the limit $h_{q}$ for $q \rightarrow 0$, and is obtained through the logarithmic averaging procedure:

$F_{0}(s) \equiv \exp \left\{\frac{1}{4 N_{s}} \sum_{v=1}^{2 N_{s}} \ln \left[F^{2}(s, v)\right]\right\} \approx s^{h_{0}} \cdot(6)$

In general the exponent $h_{q}$ will depend on $q . h_{q}$ are called as generalized Hurst exponents, due to the equivalence between $h_{2}$ and the Hurst exponent $H$ (Feder, 1988) for stationary series. If $h_{q}$ does not depend on $q$ the series is mono-fractal, while in case it is a monotonically decreasing function of $q$ the series is multifractal.

Multifractal series can be also studied by means of the singularity spectrum, obtained applying the Legendre transform (Parisi and Frisch, 1985). From the relationship

$\tau(q)=q h_{q}-1,(7)$

and

$\alpha=\frac{d \tau}{d q},(8)$

we obtain

$f(\alpha)=q \alpha-\tau(q),(9)$

where, $\alpha$ is the Hölder exponent and $f(\alpha)$ indicates the dimension of the subset of the series that is characterized by $\alpha$ (Ashkenazy et al., 2003). The multifractal spectrum indicates how much dominant 
are various fractal exponents present in the series. The width of the singularity spectrum is often used to quantitatively measure the degree of multifractality of the series, thus the wider the spectrum more multifractal the series.

\section{Results}

We analysed the magnitudes of Pannonian earthquakes occurred from 2002 to 2012 (Fig. 1a). The earthquake sequence is featured by two well distinct depth classes, shallow (depth less than $40 \mathrm{~km}$ ) and deep (depth larger than $70 \mathrm{~km}$ ) (Telesca and Toth, 2014). The two sets of events are characterized by different seismotectonic charactericstics; Fig. 1b and Fig 1c show respectively the time series of the shallow and deep earthquakes that we investigated. Before applying the MFDFA to both magnitude series, we performed the Gutenberg-Richter (GR) analysis (Gutenberg and Richter, 1944). The GR law states that $N_{(M>M t h)} \sim 10^{-b}$, where $N$ represents the number of earthquakes with magnitude larger than a threshold $M_{t h}$ and $b$ is the so called " $b$-value" that represents the proportion of small events versus large events and is given by the exponent of the power-law (the GR law for both sequences is represented by squares in Fig. 2). The power-law holds for just a limited magnitude range. The minimum of such magnitude range is called completeness magnitude, which represents the minimum magnitude over which all the earthquakes occurring in a certain area can be reliably detected. There are several methods to estimate the completeness magnitude and the $b$-value (see, for instance, van Stiphout et al., (2012) for a review). In this study, we estimated the completeness magnitude as the maximum of the binned distribution of the magnitudes (Wiemer and Wyss, 2000) (triangles in Fig. 2). For the shallow and deep catalogue the completeness magnitude $M_{c}$ is 2.5 and 3.6 respectively. The length of the shallow catalogue (considering only events with magnitude larger or equal to 2.5 ) is 2366 and that of the deep catalogue (considering only the earthquakes with magnitude larger or equal to 3.6) is 901. The $b$-value is calculated by using the maximum likelihood estimation formula by Aki (1965), 
$b=\frac{\log _{10}(e)}{<M>-\left(M_{c}-\Delta M_{b i n} / 2\right)}$

where $<M>$ is the mean magnitude of the events whose magnitude is larger than $M_{c}$, and $\Delta M_{b i n}$ is the width of the bin. The uncertainty of b-value was calculated by using the following formula (Aki, 1965): $\varepsilon_{b}=\frac{b}{\sqrt{N}}$

Therefore $\left(b, \varepsilon_{b}\right)$ for the shallow and deep catalogue are respectively $(0.82,0.02)$ and $(0.92,0.03)$. Considering only the events with magnitude larger or equal to $M_{c}$, the If the shallow catalogue is long enough, the deep catalogue is quite small, thus raising possible issues related with finite-size effects (FSE) linked with the choice of the range of scales $s$, the range of $q$ and the value of degree $m$ of the detrending polynomial.

For the analysis of the multifractal behaviour of the deep catalogue, we considered a relatively short range of the $q$ values due to the relatively short length of the series (Lòpez and Contreras, 2013). We considered q ranging between -5 and 5 with step of 0.5 and scale $s$ ranging between 10 and N/4. Fig. 3 shows the fluctuation functions corresponding to $q=-5,0$ and 5 for $m$ varying between 1 and 5 . We can observe that the fluctuations functions show a clear dependence on the minimum scale $s$ : in fact, for all the degrees $m$ at shorter scales up to approximately 16, the fluctuation functions are bent down; such effect is clearly visible for $q=-5$, weakly present for $q=0$ and apparently absent for $q=5$. Regarding the variation with the degree $m$, we can observe that for $m \geq 4$ the fluctuation functions tend to overlap, and this indicates that a 4-th degree polynomial is sufficient to remove the trends of order up to the 4-th in the profile (and so, up to the 3-rd in the series). On the base of above observations, we selected the following ranges of the parameters to calculate $h_{q}$ and the singularity spectrum: $q$ ranging between -5 
and $5, s$ between 20 and $N / 4$ and $m=4$. Fig. 4 shows the $h_{q}$ for the deep catalogue. Following Lòpez and Contreras (2013), we calculated three parameters to quantify the multifractal behaviour of the series: the mean value of the generalized Hurst exponent over all the $q\left\langle h_{q}\right\rangle$, the corresponding standard deviation $\sigma_{h}$ and the relative maximum difference between $h(q)$ and the mean $\Delta_{\max }=\left(\max \left\{h_{q}\right\}\right.$ $\left\langle h_{q}>\right) /<h_{q}>$. For the deep catalogue, these are: $\left\langle h_{q}>=0.54, \sigma_{h}=0.04\right.$ and $\Delta_{\max }=0.16$. The behaviour shown by the series appears consistent with a monofractal signal with Hurst exponent $=0.5$. Since the application of MFDFA to short series could raise some uncertainty in the results, in order to check if the values of the generalized Hurst exponents are due to the shortness of the time series or if they suggest some deeper dynamics, we applied the MFDFA-4 $(m=4)$ to two thousands Gaussian white noise series with the same length, mean magnitude and standard deviation as the original one. Then for each $q, 2000$ values were averaged and the $h_{q}$ curve was obtained (red circles in Fig. 4). The comparison between the two curves seems to suggest that the multifractality of the deep original series is not due to its shortness but is an inner dynamical feature of the series. However, in order to quantitatively test if the multifractality of the deep series is significant against the random simulations, for each random series we calculated the standard deviation $\sigma_{h, r}$ and the relative maximum difference between the Hurst exponents and the mean $\Delta_{\max , r}$. Fig. 4 shows the distribution of the $\sigma_{h, r}$ (Fig. 5a) and $\Delta_{\max , r}$ (Fig. 5b) along with the corresponding values of the deep series (red vertical arrows). It is visible that the values calculated for the deep series are located in the ending part of the distribution, indicating that their significance is high. We calculated the significance of the $\sigma_{h}$ and $\Delta_{\max }$ by using the following formula (Theiler et al., 1992)

$\sigma=\frac{\left|a-a_{R}\right|}{\sigma_{R}}$

where $a$ is $\sigma_{h}$ or $\Delta_{\max }$ and $a_{R}$ and $\sigma_{R}$ indicate the mean and the standard deviation $\sigma_{h, r}$ and $\Delta_{\max , r}$. Then the $p$-value is calculated by means of the formula $p=\operatorname{erfc}(\sigma / \sqrt{2})$; this is the probability of observing a 
significance $\sigma$ or larger if the multifractality observed in the deep sequence is obtained purely by chance. In our case we obtained $\mathrm{p}_{\sigma}=1.4 \cdot 10^{-2}$ and $\mathrm{p}_{\Delta}=8.8 \cdot 10^{-4}$; both the values are small, indicating a high significance of the $\sigma_{h}$ and $\Delta_{\max }$ respectively.

Fig. 7 shows the singularity spectrum for the deep catalogue: the singularity spectrum appears to be quite right-skewed, indicating the relative dominance of the higher generalized Hurst exponents and, then, of the small fluctuations in the magnitude series.

We applied the MFDFA-4 to the shallow series, for the same parameter ranges as the deep series. Fig. 6 shows also the comparison between the $h_{q}$ curves obtained for the two catalogues. The $h_{q}$ curve of the shallow series is shifted up with respect to the $h_{q}$ curve of the deep series, indicating a higher persistence, but the multifractal degree is lower than that of the deep series: in fact the $<h_{q}>=0.61$ $\sigma_{q}=0.03$ and $\Delta_{\max }=0.09$. Furthermore, the singularity spectrum (Fig. 7) appears more symmetric than that of the deep series. Comparing with the random series, the $p$-values of both the standard deviation and the relative maximum difference between the Hurst exponents and the mean are respectively 0.0163 and 0.0268 that indicate a significance larger than $95 \%$.

The two catalogues have different minimum magnitude, which is 2.5 and 3.6 respectively for the shallow and deep series. The found difference between the two catalogues in the multifractal character could be probably due to the different minimum magnitude that, furthermore, is directly linked with the size of the catalogue (2366 and 901 for the shallow and deep series respectively). In order to check if any change in the multifractality could occur changing the minimum magnitude, we applied the MFDFA-4 to the shallow catalogue, but varying the minimum magnitude from $2.6(N=1808)$ to 3.0 ( $N=976)$. Fig. 8 shows the comparison among the Legendre spectra: we can observe that the Legendre spectra for the magnitude series corresponding to the minimum threshold of 2.5 up to 2.8 are overlapped, while for minimum threshold of 2.9 and 3.0 they shift toward higher values of $\alpha$, indicating an increase of the persistence for these thresholds. However, the multifractal degree, linked 
with the width of the Legendre spectrum, is almost identical in all the series (see the Legendre spectra centred on the corresponding maxima in Fig. 9).

\section{Conclusions}

In this paper the multifractal properties of the earthquake magnitude sequence of Pannonian region were investigated. The comparison of the multifractal characteristics between the deep and the shallow catalogues point out to the following findings:

i) The magnitude sequence of the shallow catalogue is weakly persistent, while that of the deep one is almost uncorrelated. The uncorrelation characterizing the deep catalogue could be linked with its magnitude range: the minimum magnitude of the deep catalogue is 3.6, and this means that the catalogue is mainly formed by relatively large events, whose occurrence could probably be more random than that of the shallow events..

ii) The magnitude sequence of the deep catalogue is more multifractal than that of the shallow one. This suggests that the magnitude sequence of the deep catalogue is less homogenous than that of the shallow catalogue; this reveals that for the deep catalogue the difference between the scaling behaviour of the larger magnitude fluctuations and the smaller ones is more intense than that characterizing the shallow catalogue. The presence of more smaller magnitude events in the shallow catalogue probably tends to weaken the magnitude fluctuations, favoring the dominance of a higher homogeneity.

iii) The magnitude sequence of the deep catalogue is characterized by a right-skewed multifractal spectrum, while that of the magnitude series of the shallow one is rather symmetric. This indicates that the multifractality of the magnitude sequence of the deep catalogue is mainly governed by the small magnitude fluctuations that arise from the difference in magnitude between earthquakes. This could be probably explained by the 
higher variability of the magnitudes in the shallow catalogue than that in the deep one. The maximum magnitude in both catalogues is 5.8 , but the minimum magnitude of the deep catalogue is 3.6 (while is 2.5 in the shallow one), suggesting that the smaller magnitude variations are more dominant in the deep series than in shallow one.

iv) The overlapping effect shown by the deep catalogue with changing the minimum magnitude indicates that multifractality is invariant with respect to the magnitude threshold. Such invariance with respect to the minimum magnitude holds also for the $b$-value of the GR law $N_{(M>M t h)} \sim 10^{-b}$ (Fig. 2). Such similar behavior could suggest that probably there exists a relationship between multifractality (quantified by the width of the Legendre spectrum) and the $b$-value that could be direct, since higher the $b$-value larger the multifractality (as it could be deduced comparing the results for the deep and the shallow catalogues). However, more investigation is needed to show if a quantitative relationship exists.

\section{Acknowledgements}

L. Telesca acknowledges the financial support in the frame of the Bilateral Agreement between CNR and MTA 2013-2015.

\section{References}

Aki K (1965) Maximum likelihood estimate of $b$ in the formula $\log (N)=a-b M$ and its confidence limits.

Bull Earthq Res Inst Tokyo Univ 43:237-239.

Aggarwal, S. K., Lovallo, M., Khan, P. K., Rastogi, B. K., Telesca, L., 2015. Multifractal detrended fluctuation analysis of magnitudo series of seismicity of Kachchh region, Western India, Physica A, 426, 56-62. 
Ashkenazy, Y., Baker, D. R., Gildor, H., Havlin, S., 2003, Nonlinearity and multifractality of climate change in the past 420,000 years, Geophys. Res. Lett., 30, 2146-2149.

Bada G, Grenerczy Gy, Tóth L, Horváth F, Stein S, Cloetingh S, Windhoffer G, Fodor L. Pinter N, Fejes I, 2007. Motion of Adria and ongoing inversion of the Pannonian basin: Seismicity, GPS velocities and stress transfer. In: Stein, S., Mazzotti, S., (Eds.), Continental Intraplate Earthquakes: Science, Hazard, and Policy Issues. Geological Society of America Special Paper 425, p. 243-262, doi: $10.1130 / 2007.2425(16)$

Chamoli, A., Yadav, R. B. S., 2013. Multifractality in seismic sequences of NW Himalaya, Natural Hazards, 1-14.

Feder, J., 1988. Fractals. New York: Plenum Press

Gutenberg R, Richter CF (1944) Frequency of earthquakes in California. Bull Seism Soc Am 34:185188.

Kantelhardt, J., Zschiegner, S., Koscielny-Bunde, E., Bunde, A., Havlin, S., Stanley, E., 2002, Multifractal detrended uctuation analysis of nonstationary time series, Physica A, 316, 87-114

Lennartz, S., Livina, V. N., Bunde, A., Havlin, S., 2008. Long-term memory in earthquakes and the distribution of interoccurrence times, Europhys. Lett., 81, 69001

López, J. L., Contreras, J. G., 2013. Performance of multifractal detrended fluctuation analysis on short time series, Phys. Rev. E, 87, 022918

Parisi, G., Frisch, U., 1985, On the singularity structure of fully developed turbulence. In Turbulence and Predictability in Geophysical Fluid Dynamics (eds. M. Ghil, R. Benzi and G. Parisi). Proceedings of the International School of Physics "Enrico Fermi" (1983), 84-87, NorthHolland.

Shi, Y., Bolt, B. A. (1982), The standard error of the magnitude-frequency b-value, Bull. Seismol. Soc. Am., 72, 1677-1687. 
Telesca, L., Babayev, G., Kadirov, F., 2012a. Temporal clustering of the seismicity of the AbsheronPrebalkhan region in the Caspian Sea area, Nat. Hazards Earth Syst. Sci., 12, 3279-3285.

Telesca, L., Cuomo, V., Lapenna, V., Macchiato, M., 2001. Intermittent-type temporal fluctuations in seismicity of the Irpinia (southern Italy) region, Geophys. Res. Lett., 28, 3765-3768.

Telesca, L., Cuomo, V., Lapenna, V., Macchiato, M., 2002a. On the methods to identify clustering properties in sequences of seismic time-occurrences, J. Seismology, 6, 125-134.

Telesca, L., Lapenna, V., 2006. Measuring multifractality in seismic sequences, Tectonophysics, 423, 115-123.

Telesca, L., Lapenna, V., Vallianatos, F., 2002b. Monofractal and multifractal approaches in investigating scaling properties in temporal patterns of the 1983-2000 seismicity in the Western Corinth Graben (Greece), Phys. Earth Planet. Int., 131, 63-79.

Telesca, L., Lovallo, M., Toth, L., 2014. Visibility graph analysis of 2002-2011 Pannonian seismicity, Physica A, 416, 219-224.

Telesca, L., Mohamed, A. E.-E. A., ElGabry, M., El-hady, S., Abou Elenean, K. M., 2012b. Time dynamics in the point process modelling of seismicity of Aswan area (Egypt), Chaos Solitons \& Fractals, 45, 47-55.

Theiler, J., Eubank, S., Longtin, A., Galdrikian, B., Farmer, J. D., 1992. Testing for nonlinearity in time series: the method of surrogate data, Physica D 58, 77-94

Tóth, L., Győri, E., Mónus, P., Zsíros, T., 2006, Seismic Hazard in the Pannonian Region. In: Pinter, N., Grenerczy, Gy., Weber, J., Stein, S., Medak, D., (eds.), The Adria Microplate: GPS Geodesy, Tectonics, and Hazards. Springer Verlag, NATO ARW Series, Vol. 61, p. 369-384

Tóth, L., Mónus, P., Zsíros, T., Kiszely, M., 2002, Seismicity in the Pannonian Region - earthquake data, EGU Stephan Mueller Special Publication Series, 3, 9-28 
van Stiphout, T., J. Zhuang, and D. Marsan (2012), Seismicity declustering, Community Online Resource for Statistical Seismicity Analysis, doi:10.5078/corssa-52382934. Available at http://www.corssa.org.

Varotsos, P. A., Sarlis, N. V., Skordas, E. S., 2012. Scale-specific order parameter fluctuations of seismicity before mainshocks: Natural time and Detrended Fluctuation Analysis, Europhys. Lett., $99,59001$.

Wiemer S, Wyss M (2000) Minimum Magnitude of Completeness in Earthquake Catalogs: Examples from Alaska, the Western United States, and Japan. Bull Seismol Soc Am 90:859-869. 


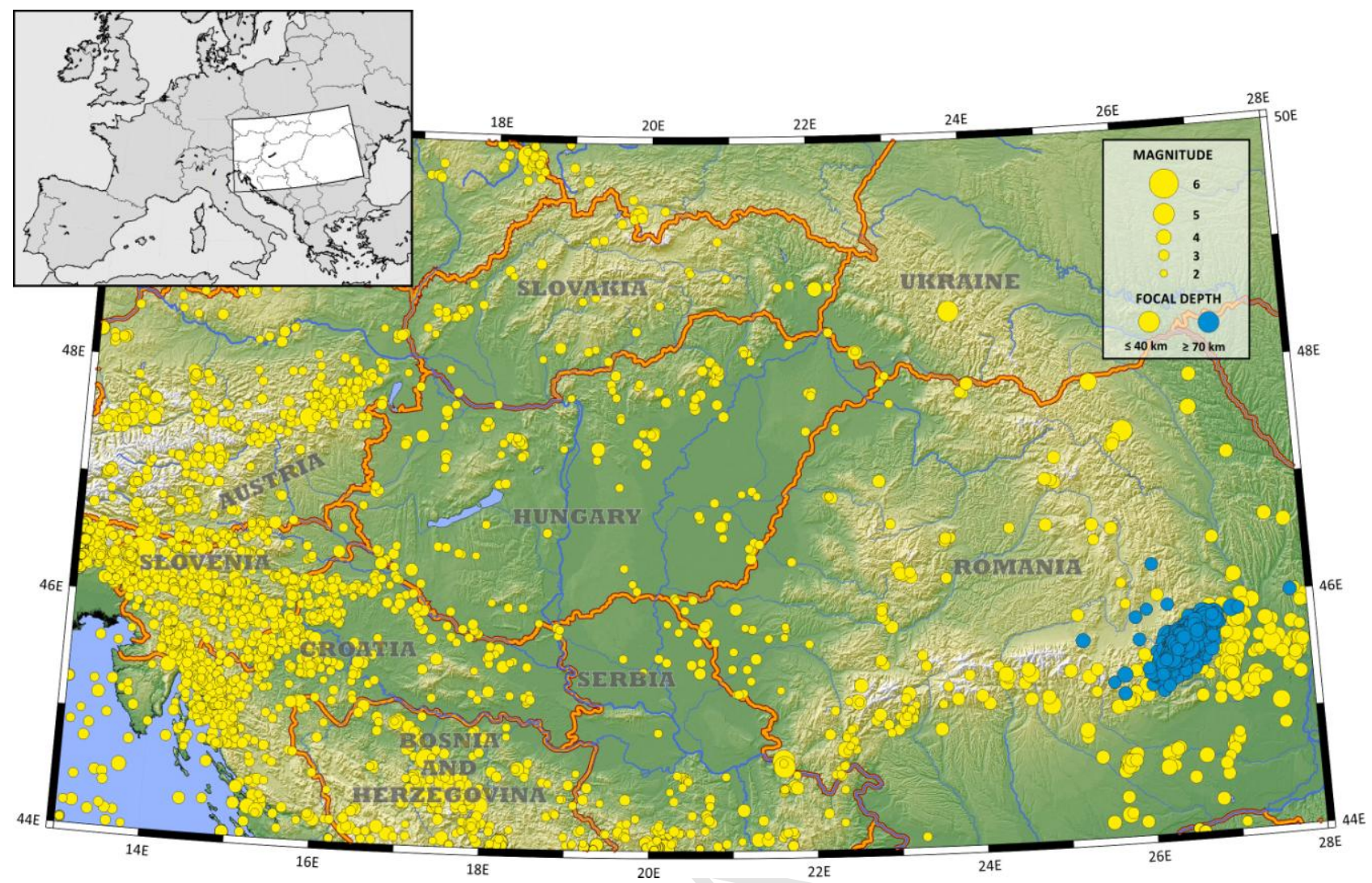

a)

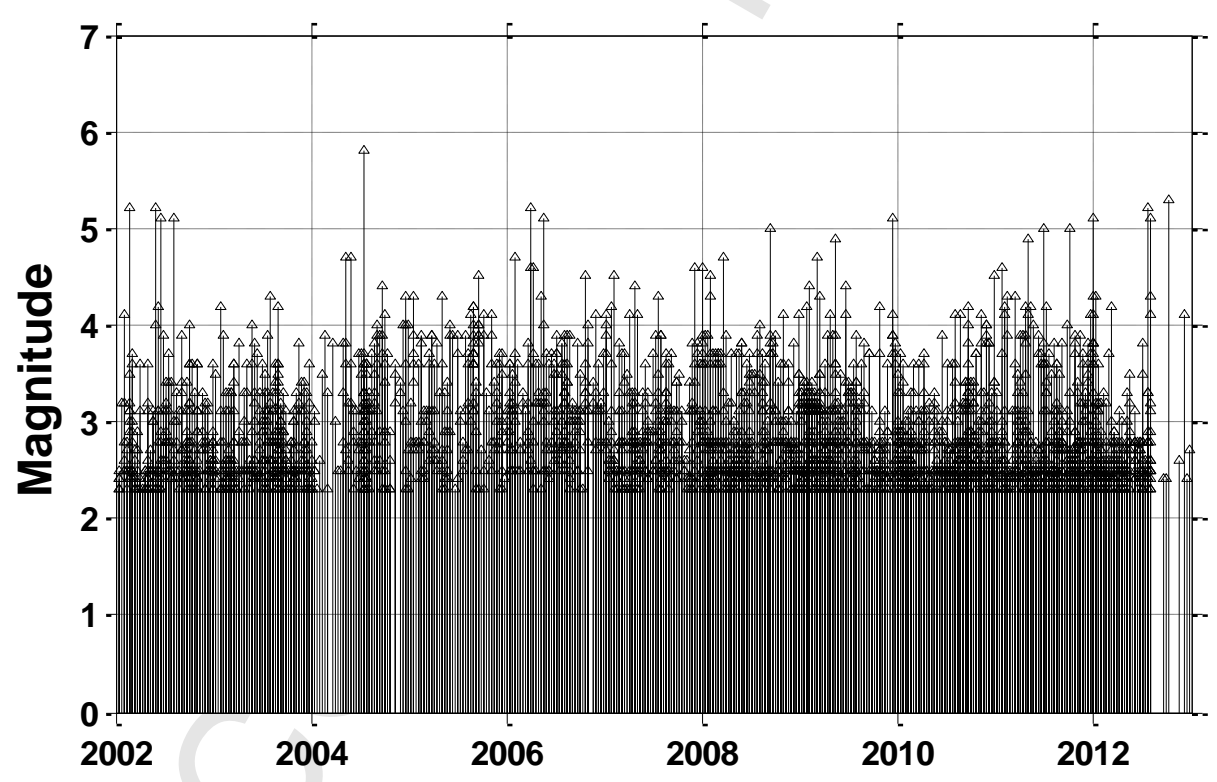

b) 


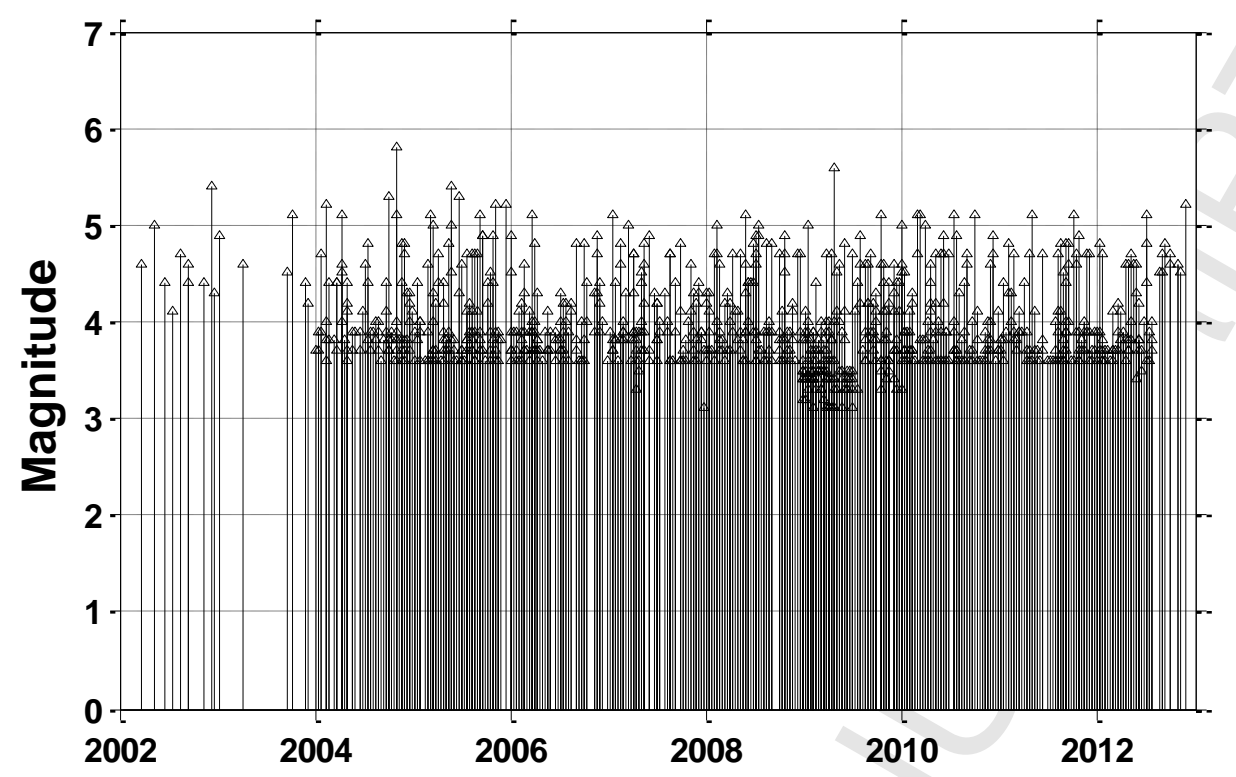

c)

Fig. 1. a) Spatial distribution of the Pannonia earthquakes: the shallow (depth smaller than $40 \mathrm{~km}$ ) events are indicated by yellow circles; the deep (depth larger than $70 \mathrm{~km}$ ) events by blue circles; b) magnitude time series of shallow earthquakes; c) magnitude time series of deep earthquakes.
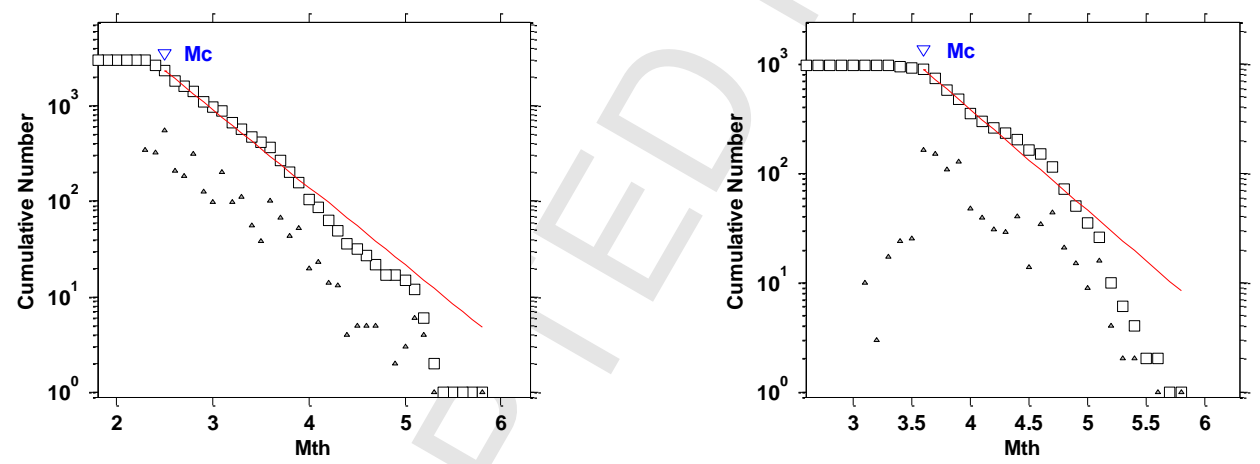

a)

b)

Fig. 2. Magnitude distribution of the shallow catalogue (a) and deep catalogue (b). The triangles indicate the binned magnitude distribution, while the squares indicate the cumulative number of earthquakes with magnitude larger than the $M_{t h}$. The $b$-value of the GR law, estimated by using the MLE method, is 0.82 for the shallow series and 0.92 for the deep one. The red lines represent the GR law corresponding to the estimated $b$-value. 

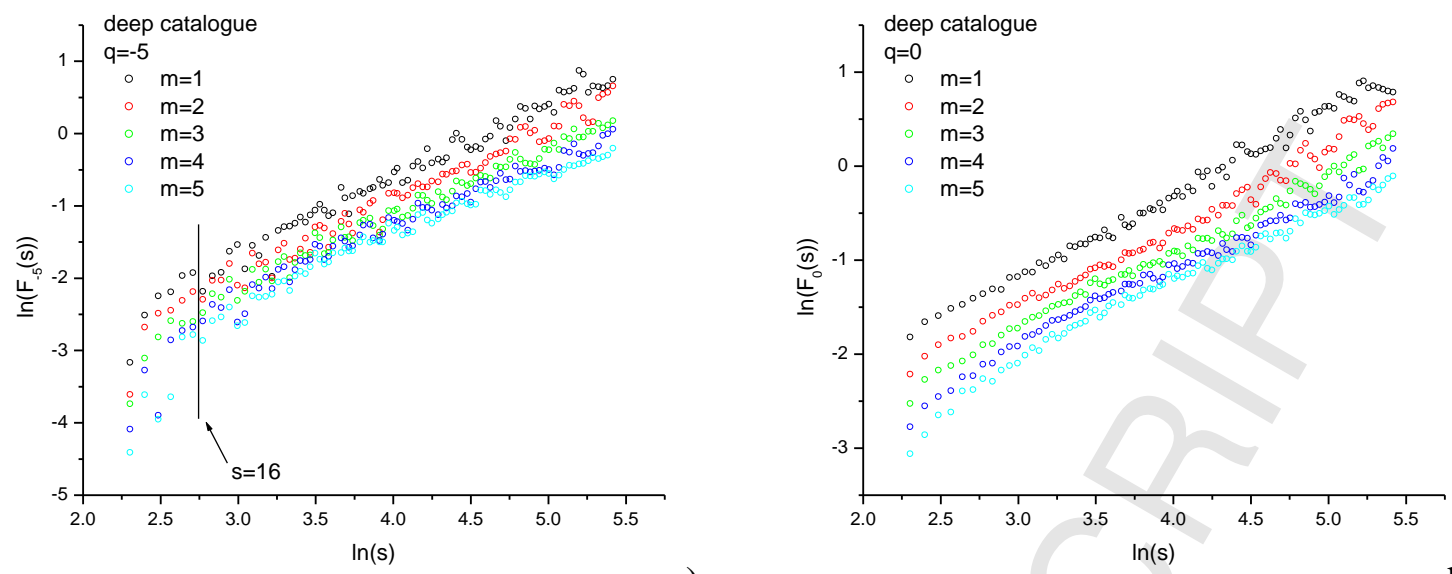

a)

b)

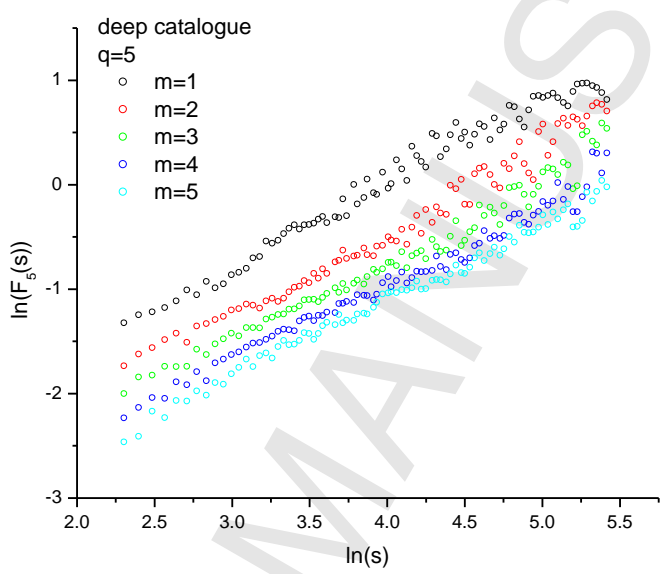

c)

Fig. 3. Fluctuation functions of the deep catalogue for $q=-5$ (a), $q=0$ (b) and $q=5$ (c), for several degrees of the detrending polynomial $m$.

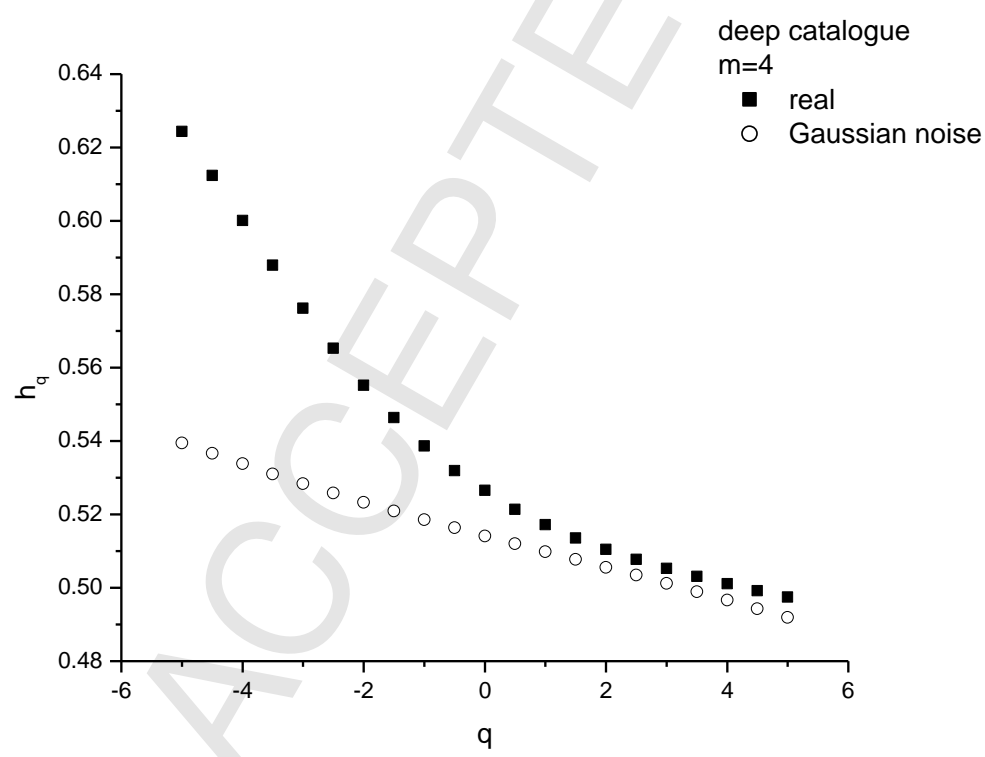

Fig. 4. $h_{q}$ for the deep catalogue (black squares) and average over 2000 realizations of Gaussian noise. 


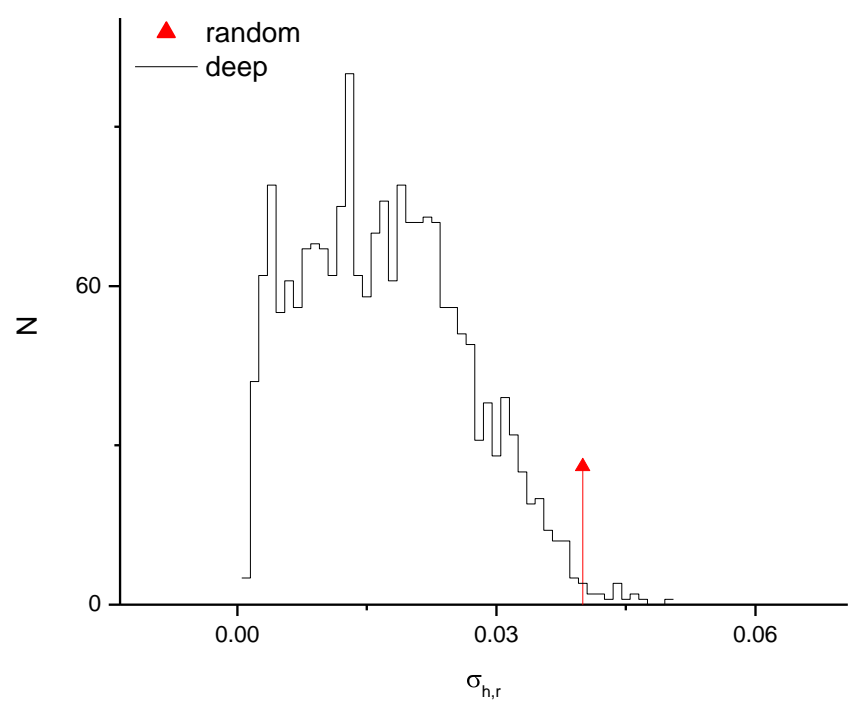

a)

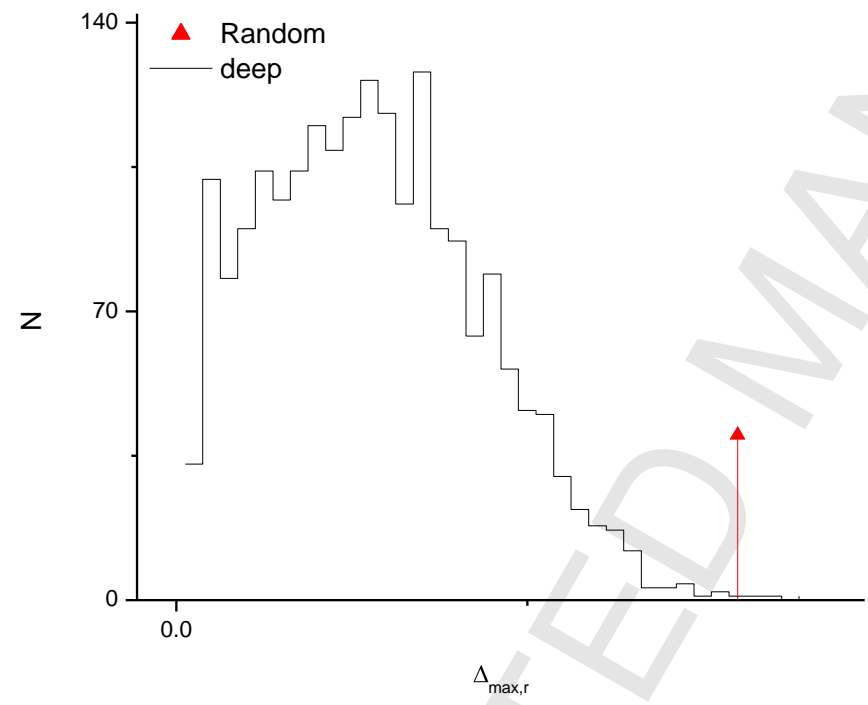

b)

Fig. 5. Distribution of the standard deviation (a) and relative maximum difference between the Hurst exponents and the mean for the Gaussian realization. The red vertical arrows indicate the values for the deep series. 


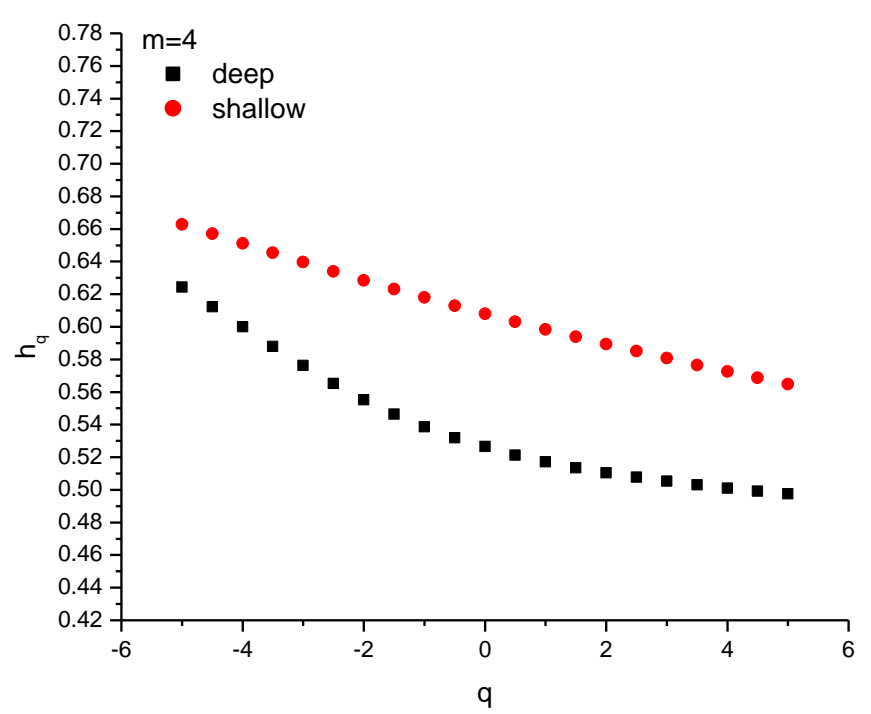

Fig. 6. Generalized Hurst exponents for the deep (black squares) and shallow (red circles) catalogues.

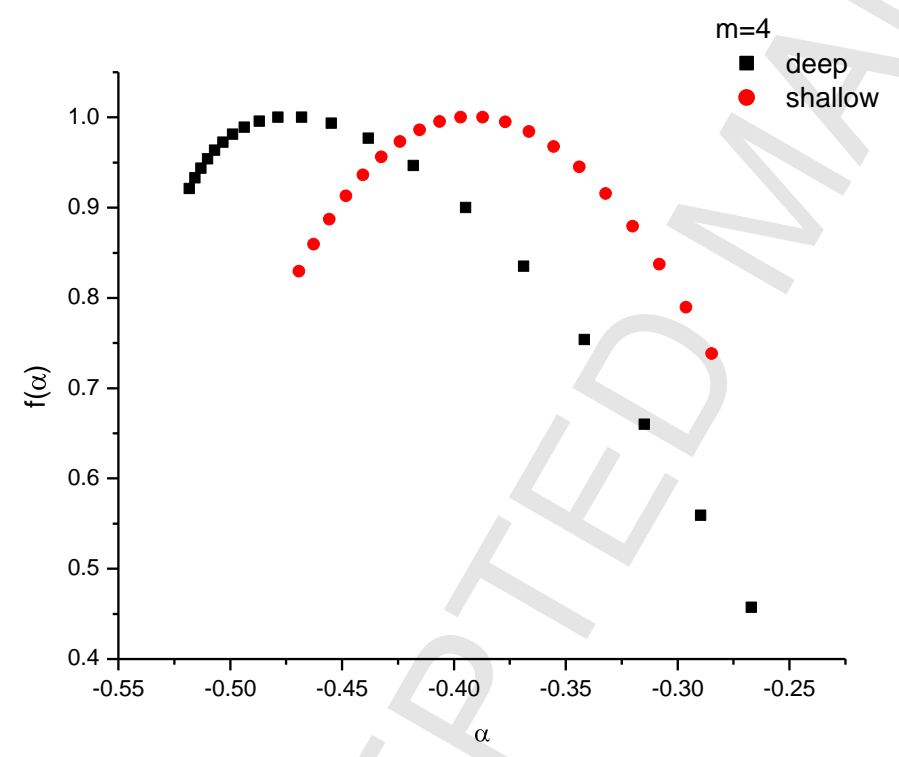

Fig. 7. Singularity spectrum for the deep (black squares) and shallow (red circles) catalogues. 


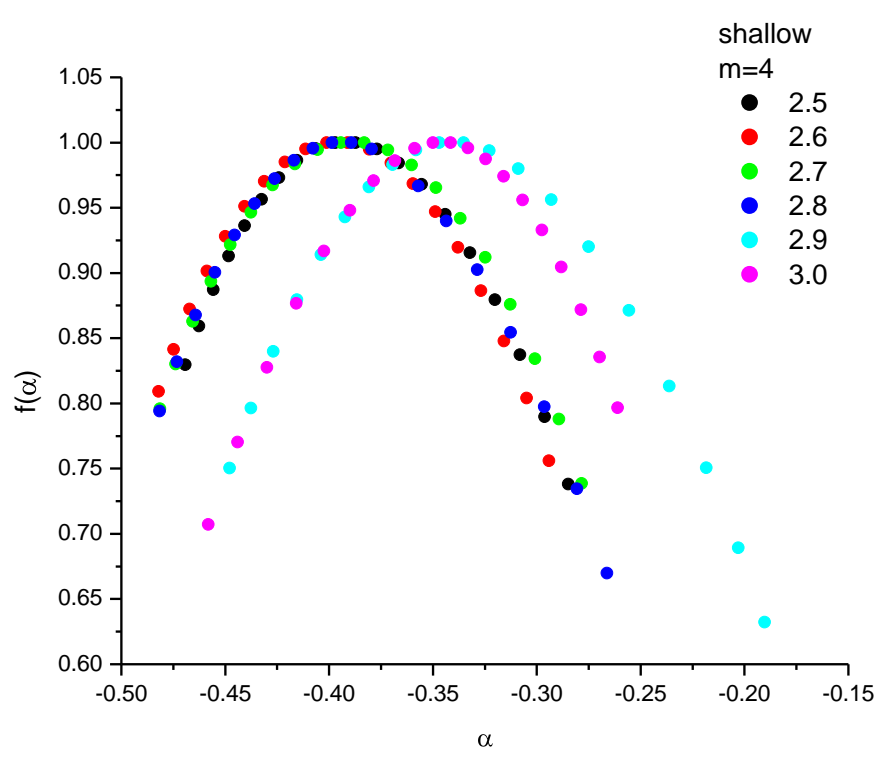

Fig. 8. Comparison among the singularity spectra of the shallow catalogue for different minimum magnitude.

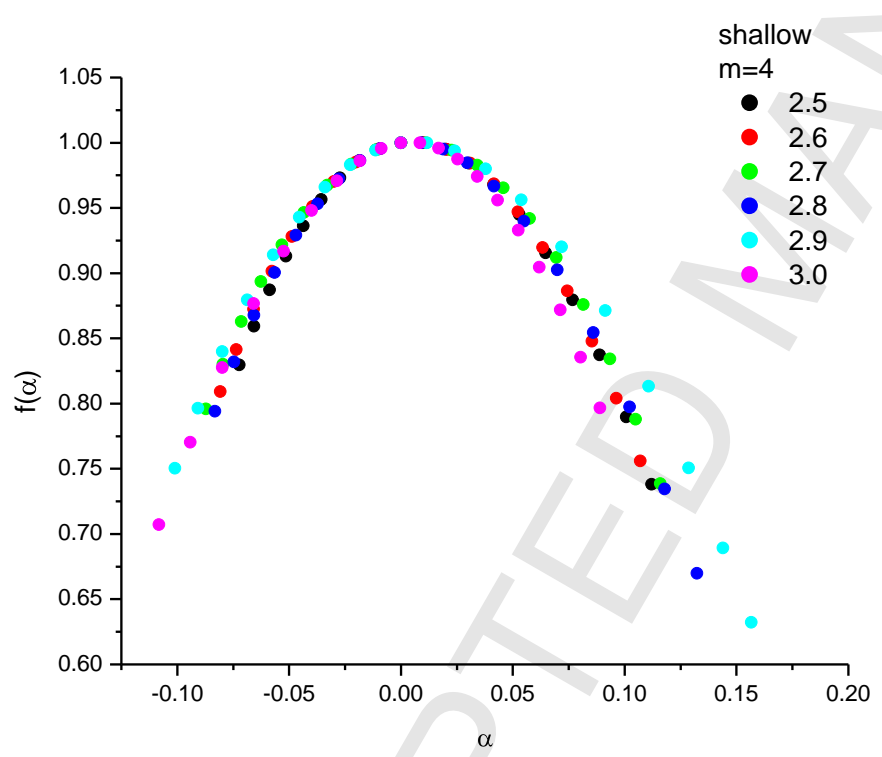

Fig. 9. Comparison among the singularity spectra of the shallow catalogue centred on the corresponding maxima. 